\title{
HoNgos ARBUSCULARES (GLOMEROMYCOTA) EN LA RIZOSFERA DE ATRIPLEX LAMPA EN dOS AMBIENTES SALINOS De CóRDOBa: INFLUENCIA DE LA PROFUNDIDAD EN LA COLONIZACIÓN RADICAL Y PRESENCIA DE MORFOESPECIES
}

\author{
FLORENCIA SOTERAS 1 , NOELIA COFRÉ1, JOSÉ BARTOLONI², MARTA CABELLO ${ }^{3} y$ \\ ALEJANDRA BECERRA ${ }^{1}$
}

\begin{abstract}
Summary: Arbuscular fungi (Glomeromycota) in the rhizosphere of Atriplex lampa at two saline environments of Córdoba (Argentina): depth influence on root colonization and the presence of morphospecies. Atriplex lampa is a valuable fodder shrub available for browsing by livestock even during drought periods in the Chaco Phytogeographical Province. Halophytes may benefit from the association with arbuscular mycorrhizal fungi (AMF) through improved tolerance to drought and salt. Ecological studies of AMF are generally restricted to the main rooting zone. However, AMF vertical distribution and seasonal dynamics in natural saline soils of Argentina have been poorly studied. The aim of this work was to explore AMF root colonization, root concentration and to identify AMF morphoespecies in $A$. lampa rhizosphere in two saline environments (Salinas de Ambargasta and Salinas Grandes) of central Argentina, in five soil depth levels, during the wet and dry seasons. Despite we did not find arbuscules, AMF were found colonizing A. lampa roots in all depth levels. Salinas Grandes showed the highest root colonization value, and showed the highest root concentration, during wet season. The 20 AMF morphospecies identified in this work belonged to the genera: Acaulospora, Ambispora, Claroideoglomus, Diversispora, Funneliformis, Glomus, Septoglomus and Scutellospora. This is the second record in Argentina of AMF structures in A. lampa roots. Future studies that evaluate mycorrhizal dependency of the plant are necessary to confirm the function of the symbiosis.
\end{abstract}

Key words: Arbuscular mycorrhizal fungi; saline soils; Atriplex lampa; depth soil; seasonality

Resumen: Atriplex lampa es un arbusto halófito de la provincia fitogeográfica Chaqueña que constituye un recurso forrajero a lo largo de todo el año. Las plantas halófitas se pueden beneficiar al asociarse con los hongos micorrícico arbusculares (HMA), ya que le proveen resistencia contra la salinidad y la sequía. En general, los estudios acerca de los HMA están restringidos a los primeros centímetros del suelo y existe poca información sobre su presencia a mayores profundidades. El objetivo de este trabajo fue estudiar la colonización, la concentración radical e identificar las morfoespecies de HMA en la rizosfera de A. lampa en dos ambientes salinos del norte de la provincia de Córdoba, durante dos estaciones del año, y en cinco profundidades del suelo. Aunque no se encontraron arbúsculos, $A$. lampa presentó colonización radical por HMA en todas las profundidades. El mayor valor de colonización micorrícica y de concentración radical se observó en las Salinas Grandes. Se identificaron 20 morfoespecies de HMA pertenecientes a los géneros: Acaulospora, Ambispora, Claroideoglomus, Diversispora, Funneliformis, Glomus, Septoglomus y Scutellospora. Este es el segundo registro en Argentina de la presencia de HMA en raíces de $A$. lampa. Es necesario realizar estudios que evalúen la respuesta de $A$. lampa frente a la inoculación con HMA para confirmar la funcionalidad de la simbiosis.

Palabras clave: Hongos micorrícico arbusculares; suelos salinos; Atriplex lampa; profundidad del suelo, estacionalidad.

\footnotetext{
${ }^{1}$ Laboratorio de Micología, Instituto Multidisciplinario de Biología Vegetal (IMBIV) - CONICET- Universidad Nacional de Córdoba (UNC)- CC 495, 5000 Córdoba, Argentina. TE 54-0351-433-4141/4404/4408 int 444, fsoteras@conicet.gov.ar.

${ }^{2}$ Cátedra de Métodos Cuantitativos Aplicados, Facultad de Agronomía, Universidad de Buenos Aires (UBA). Tel-Fax: $54-$ 011-45248000, República Argentina.

${ }^{3}$ Instituto Spegazzini. Facultad de Ciencias Naturales y Museo. Avenida 53, № 477. 1900 La Plata. Tel-Fax: 54-02214219845, República Argentina.
} 


\section{INTRODUCCIÓN}

Los suelos salinos ocupan aproximadamente el $7 \%$ de la superficie de la tierra (Ruiz-Lozano et $a l ., 1996)$. En el norte de la provincia de Córdoba, estos suelos son de origen natural y ocupan el $14 \%$ del área total (Cabido \& Zak, 1999). En estos ambientes, la vegetación se distribuye en relación a la concentración de sales en el suelo y las halófitas, la mayoría pertenecientes a la familia Chenopodiaceae, representan el $9 \%$ de la cobertura vegetal. Las plantas halófitas poseen mecanismos morfo-fisiológicos que les permiten aprovechar de manera eficiente los escasos recursos nutricionales de los suelos salinos (Khan \& Duke, 2001) y, en particular, su asociación con los hongos micorrícico arbusculares (HMA) les provee tolerancia a la salinidad y a la sequía (Smith $\&$ Read, 2008).

En la literatura, la familia Chenopodiaceae ha sido registrada como no susceptible a la colonización micorrícica (Hirrel et al., 1978), sin embargo numerosos estudios han reportado que los géneros Salicornia, Suaeda, Atriplex y Salsola presentan colonización por HMA (Allen, 1983; Allen \& Allen, 1990; Sengupta \& Chaudhuri, 1990; Aguilera et al., 1998; Hildebrandt et al., 2001; Plenchette \& Duponnois, 2005; Cofré et al., 2012). Según Sengupta \& Chaudhuri (1990), es el estrés provocado por la presencia de sales en los suelos, lo que hace más susceptible a las quenopodiáceas a ser colonizadas por los HMA.

Atriplex lampa (Moq.) D. Dietr. ("zampa") es un arbusto que constituye un buen recurso forrajero todo el año, siendo palatable para ovinos y caprinos incluso en períodos críticos de sequía o de escasez de alimento (Passera \& Borsetto, 1989). Aunque los antecedentes bibliográficos referidos a $A$. lampa en Argentina son escasos, ya se ha reportado la presencia de HMA en sus raíces (Fontenla et al., 2001).

Los estudios ecológicos sobre los HMA, usualmente están restringidos a los primeros 20 centímetros del suelo, donde se encuentra la mayor biomasa radical (Brundrett, 1991), y pocos trabajos incluyen capas más profundas del suelo. En general, se ha observado una disminución en la colonización micorrícica (Rillig \& Field, 2003), en el número de propágulos infectivos (esporas, hifas y raíces micorrizadas) (An et al., 1990) y en la cantidad de micelio extrarradical (Kabir et al., 1998) al aumentar la profundidad del suelo. Por otro lado, la dinámica estacional de la colonización micorrícica ha sido reportada en muchos estudios (Carvalho et al., 2001; Lugo et al., 2003; Füzy et al., 2008). Sin embargo, aún es escasa la información sobre los HMA en suelos semiáridos de ambientes naturales (Wang et al., 2004).

El objetivo de este trabajo fue estudiar en dos ambientes salinos del norte de Córdoba y en dos estaciones del año (verano/estación húmeda e inverno/estación seca), la colonización micorrícica y la concentración radical (número de segmentos radicales en $100 \mathrm{~cm}^{3}$ de volumen de suelo) e identificar las morfoespecies de HMA presentes en la rizosfera de A. lampa en cinco profundidades del suelo.

\section{Material y Método}

\section{Sitios de estudio}

El estudio se realizó en dos zonas del norte de la provincia de Córdoba, las Salinas de Ambargasta (29 27' 45,6" S , 64 $4^{\circ} 18^{\prime} 21,9^{\prime \prime} \mathrm{W}$ ) perteneciente al Refugio de Vida Silvestre "Monte Las Barrancas" y las Salinas Grandes (29 44' 12,5” S, 64 31' 46,4 " W) en la Reserva de Uso Múltiple "Salinas Grandes" (Departamentos de Tulumba, Ischilín y Cruz del Eje). Las escasas precipitaciones (menos de $500 \mathrm{~mm}$ anuales), junto a la temperatura media anual de $19,9^{\circ} \mathrm{C}$ determinan un clima cálido y seco, perteneciente al Dominio Semidesértico. La vegetación está determinada por un gradiente de concentración salina desde la planicie occidental al interior de las depresiones salinas. En la zona más elevada predomina el bosque xerófilo bajo y abierto con un estrato arbustivo formado principalmente por especies halófitas. En contacto con la depresión salina, se desarrolla un matorral halófilo bajo con arbustos dominantes pertenecientes a la familia Chenopodiaceae: Allenrolfea patagonica (Moq.) Kuntze (jume) y Heterostachys ritteriana (Moq.) Moq. (jume), acompañados por subarbustos halófitos, tales como Atriplex argentina Speg. (cachiyuyo), A. lampa (Moq.) D. Dietr. (zampa), Salicornia ambigua (jume) y Suaeda divaricata Moq. (jume), y arbustos subhalófilos como Cyclolepis genistoides D. Don., Prosopis strombulifera (Lam.) Benth. y Maytenus vistis-idaea Griseb. (Cabido \& Zak, 1999; Cabido et al., 2006). 


\section{F. Soteras et al. - Glomeromycota: presencia y colonización radical en Atriplex lampa}

\section{Muestras de raices}

La extracción de muestras de raíces se realizó en los dos sitios durante la estación húmeda (Marzo 2007) y la estación seca (Agosto 2008). En cada sitio, durante cada estación se eligieron al azar 5 individuos de $A$. lampa, separados entre sí por una distancia mayor a $50 \mathrm{~m}$. De la rizosfera de cada individuo, se extrajeron con un sacabocado ( $3 \mathrm{~cm}$ de diámetro) una muestra de raíces y suelo a diferentes profundidades (0-10 $\mathrm{cm}, 10-20 \mathrm{~cm}, 20-30 \mathrm{~cm}, 30-40 \mathrm{~cm}$ y $40-50 \mathrm{~cm}$ ), que se guardó en bolsas de polietileno y conservó en heladera a $4^{\circ} \mathrm{C}$ hasta su posterior estudio en laboratorio.

\section{Análisis de suelo}

A fin de caracterizar el suelo de cada sitio, a las 5 muestras tomadas por nivel de profundidad se les midieron los siguientes parámetros edáficos: conductividad eléctrica $(\mathrm{mmhos} / \mathrm{cm})$, fósforo extraíble determinado por el método de Bray and Kurtz I (Jackson, 1964), pH, contenido de materia orgánica siguiendo la metodología de Nelson \& Sommers (1982), relación carbono: nitrógeno y textura del suelo. El nitrógeno total fue determinado usando el método de microKjeldhal (Bremner \& Mulvaney, 1982).

\section{Colonización y concentración radical}

Una vez en el laboratorio y en bandejas con agua, se separaron las raíces de $A$. lampa y se fijaron en FAA (formol: alcohol: ácido acético) hasta su posterior estudio. La presencia de colonización micorrícica en las raíces fue evidenciada mediante la técnica de Phillips \& Hayman (1970). El porcentaje de longitud de raíz colonizada se cuantificó mediante el método de "intersección con la cuadrícula" (Giovannetti \& Mosse, 1980), analizando 100 segmentos de $1 \mathrm{~cm}$ de longitud bajo lupa estereoscópica Leica M 420. Posteriormente, en 20 segmentos colonizados de cada muestra se observaron las estructuras intracelulares (circunvoluciones, arbúsculos, vesículas, hifas y puntos de entrada) (Ocampo et al., 1980) bajo microscopio óptico Leica 4-100X. Para cada profundidad muestreada, se cuantificó la concentración radical (número de segmentos radicales de $1 \mathrm{~cm}$ de longitud concentrados en $100 \mathrm{~cm}^{3}$ de volumen de suelo). La colonización micorrícica se clasificó según las categorías propuestas por Zangaro et al. (2002): muy alta $(>80 \%)$, alta $(60-79 \%)$, media $(40-59 \%)$, baja (20-39\%) y muy baja (1-19\%).

Morfoespecies de HMA de la rizosfera de A. lampa

Las esporas de los HMA se extrajeron de las muestras de suelo tomadas de la rizosfera de $A$. lampa en cada sitio. De cada muestra se tomaron $100 \mathrm{~g}$, que fueron tamizados empleando mallas de distinto tamaño de apertura (125 y 38 $\mu \mathrm{m})$, siguiendo la metodología propuesta por Gerdemann \& Nicolson (1963). Posteriormente, los contenidos retenidos en los tamices se centrifugaron en gradiente de sacarosa al 80 \% (Walker et al., 1982). Las esporas obtenidas se colocaron en cápsulas de Petri de $9 \mathrm{~cm}$ de diámetro con base cuadriculada de $1 \mathrm{~cm}$ de lado.

Las esporas y esporocarpos (aparentemente saludables y sin ningún tipo de ataque) fueron identificados a nivel de especie, bajo microscopio estereoscópico y microscopio óptico. Para cada tipo morfológico, se realizaron preparados permanentes usando Polivinil-alcohol (PVA) y PVA + Melzer (Omar et al., 1979), como medios de montaje. Los especímenes se identificaron bajo microscopio siguiendo los manuales de Schenk \& Perez (1990), INVAM, Krüger et al. (2012) y Oehl et al. (2011).

\section{Análisis estadísticos}

Considerando que se tomaron 5 muestras sobre la misma planta $(0-10,10-20,20-30,30-40$, y 40-50 cm), se realizó un análisis de la varianza (ANOVA) de medidas repetidas para evaluar el efecto del nivel de profundidad (factor intrasujeto), la estación y el sitio (factores inter-sujeto) en los parámetros del suelo, la colonización radical y la concentración radical. Cuando las medidas no fueron independientes (según el test de esfericidad de Mauchly), se realizó un análisis multivariado de la varianza (MANOVA). Se aplicó el test a post hoc de Tukey HSD con un nivel de significancia de 0,05 para la comparación entre medias. Además se realizaron correlaciones de Spearman entre la colonización radical y la concentración radical. Todos los análisis se realizaron utilizando el programa estadístico STATISTICA. 


\section{Resultados}

\section{Características del suelo}

Los suelos de las Salinas de Ambargasta (SA) y las Salinas Grandes (SG) son Salortides típicos (Jarsún et al., 2003). Tanto los suelos de las SA, como los de las SG, presentaron una textura franco arcillo arenosa, alto $\mathrm{pH}$, elevada conductividad eléctrica y bajo contenido de materia orgánica. De acuerdo a su pH, los suelos de las SA son fuertemente alcalinos; mientras que los de las SG son moderadamente alcalinos. El nivel de $\mathrm{P}$ fue muy bajo $(<5 \mathrm{ppm})$ en los suelos de las SA y bajo (5-12 ppm) en los de las SG. Algunos parámetros del suelo se vieron influenciados significativamente por la profundidad del suelo. En ambos sitios pudo observarse una disminución de la materia orgánica, sólo significativa en las SA, y un incremento de la conductividad eléctrica con la profundidad del suelo, aunque no significativo. Además la relación $\mathrm{C} / \mathrm{N}$ en las $\mathrm{SA}$ fue significativamente menor de $0-10 \mathrm{~cm}$ que en la capa más profunda $(40-50 \mathrm{~cm})$. En las SG este aumento de la relación $\mathrm{C} / \mathrm{N}$ con la profundidad del suelo no fue significativa (Tabla 1).

\section{Colonización y concentración radical}

Las raíces de $A$. lampa presentaron colonización micorrícica del tipo morfológico Paris. A pesar de que no se observaron arbúsculos, se encontraron puntos de entrada; hifas intracelulares de 3,2$12,1 \mu \mathrm{m}$ de diámetro, ramificadas, de pareces delgadas; circunvoluciones intracelulares y vesículas intracelulares, ovales a alargadas de 14,4$37 \mu \mathrm{m}$ de diámetro, de paredes delgadas (Fig. 1).

Las raíces de $A$. lampa presentaron colonización en todas las profundidades del suelo, en ambos sitios y durante las dos estaciones. El porcentaje de raíz colonizada varió desde muy bajo a muy alto $(4,76 \%$ a $82,35 \%)$ y fue significativamente mayor en las SG (49,02\%) que en las SA (30,31\%) (Tabla $2)$. No se observaron diferencias significativas entre las estaciones ni entre las profundidades del suelo (Tabla 2).

La concentración radical disminuyó significativamente con la profundidad (estación húmeda, $F=14,92 ; P<0,005$; estación seca, $F$ $=6,12 ; P<0,05$ ) (Fig. 2). Se observó una triple interacción entre profundidad $\mathrm{x}$ estación $\mathrm{x}$ sitio (Tabla 3), las SG presentaron la mayor concentración radical en las primeras dos capas del suelo $(0-10 \mathrm{~cm}$ y $10-20 \mathrm{~cm})$ y, a su vez estas diferencias fueron mayores durante la estación húmeda.

Las correlaciones de Spearman entre la colonización radical y la concentración radical no fueron significativas para ninguno de los sitios $(r=$ 0,$02 ; P=0,91$ y $r=0,14 ; P=0,32$ para las SA y las $\mathrm{SG}$, respectivamente).

Morfoespecies de HMA de la rizosfera de A. lampa

En los dos sitios y durante las dos estaciones, se identificaron 20 morfoespecies de HMA pertenecientes a ocho géneros (Acaulospora, Ambispora, Claroideoglomus, Diversispora, Funneliformis, Glomus, Septoglomus y Scutellospora), siendo Glomus. el más frecuente ( 90 $\%$ del total de especies). De las 20 morfoespecies, trece pudieron identificarse a nivel de especie: Acaulospora bireticulata Rothwell \& Trappe, A. scrobiculata Trappe, A. aff. undulata Sieverd., Ambispora leptoticha (N.C. Schenck \& G.S. Sm.) C. Walker, Vestberg \& A. Schüßler, Claroideoglomus etunicatum (Becker \& Gerd.) C. Walker \& A. Schüßler, C. luteum (L.J. Kenn., J.C. Stutz \& J.B. Morton) C. Walker \& A. Schüßler, Diversispora spurca (C.M. Pfeiff. et al.) C. Walker \& A. Schüssler, Funneliformis geosporum (T.H Nicolson \& Gerd.) C. Walker \& A. Schüßler, F. mosseae (T.H Nicolson \& Gerd.) C. Walker \& A. Schüßler, Glomus brohultii Sieverd. \& Herrera, G. clarum T.H. Nicolson \& N.C. Schenck, G. magnicaule I. R Hall y Septoglomus aff. constrictum (Trappe) Sieverd., G.A. Silva \& Oehl.

\section{Discusión}

En este estudio Atriplex lampa presentó colonización micorrícica, al igual que otras especies del género (Allen, 1983; Aguilera et al., 1998; Fontenla et al., 2001; Plenchette \& Duponnois, 2005; Cofré et al., 2012). No existen registros de la morfología de la colonización radical por HMA en la familia Chenopodiaceae. De acuerdo a las características morfológicas observadas (circunvoluciones, hifas y vesículas intracelulares), A. lampa presentó el tipo morfológico Paris (Peterson et al., 2004). Tal como lo mencionan Plenchette \& Duponnois (2005) y Smith \& Smith (2011), probablemente las especies de Atriplex sp. establezcan una simbiosis funcional con los 


\section{F. Soteras et al. - Glomeromycota: presencia y colonización radical en Atriplex lampa}

Tabla 1. Propiedades del suelo de las Salinas de Ambargasta (SA) y las Salinas Grandes (SG), a cinco profundidades de suelo $(0-10,10-20,20-30,30-40$, y $40-50 \mathrm{~cm})$. Los valores corresponden al promedio de 5 muestras \pm error estándar. Los valores dentro de cada fila con letras distintas son significativamente diferentes según el test de Tukey HSD para un $P<0,05$.

\begin{tabular}{|c|c|c|c|c|c|c|}
\hline & & $0-10 \mathrm{~cm}$ & $10-20 \mathrm{~cm}$ & $20-30 \mathrm{~cm}$ & $30-40 \mathrm{~cm}$ & $40-50 \mathrm{~cm}$ \\
\hline \multirow{2}{*}{ MO ${ }^{1}(\%)$} & SA & $1,29 \pm 0,40 a$ & $0,81 \pm 0,18 a b$ & $0,82 \pm 0,17 \mathrm{ab}$ & $0,71 \pm 0,21 b$ & $0,65 \pm 0,23 b$ \\
\hline & SG & $2,30 \pm 1,93 a$ & $1,41 \pm 1,06 a$ & $1,04 \pm 0,61 a$ & $1,05 \pm 0,71 \mathrm{a}$ & $0,86 \pm 0,46 a$ \\
\hline \multirow{2}{*}{$C / N^{2}$} & SA & $8,24 \pm 1,72 a$ & $7,90 \pm 0,62 a$ & $10,26 \pm 2,57 a b$ & $10,47 \pm 2,88 a b$ & $12,93 \pm 2,59 b$ \\
\hline & SG & $9,79 \pm 2,35 a$ & $9,23 \pm 2,48 a$ & $10,91 \pm 3,63 a$ & $11,95 \pm 2,04 a$ & $12,52 \pm 0,65 a$ \\
\hline \multirow{2}{*}{ Fósforo (ppm) } & SA & $11,61 \pm 6,74 a$ & $10,89 \pm 7,93 a$ & $8,53 \pm 5,55 a$ & $8,76 \pm 5,65 a$ & $9,17 \pm 4,81 \mathrm{a}$ \\
\hline & SG & $7,04 \pm 3,39 a$ & $6,48 \pm 2,68 a$ & $6,60 \pm 3,40 a$ & $7,17 \pm 4,15 a$ & $9,93 \pm 4,40 a$ \\
\hline \multirow{2}{*}{ pH 1: 2,5} & SA & $8,17 \pm 0,57 a$ & $8,11 \pm 0,50 a$ & $8,07 \pm 0,42 a$ & $8,02 \pm 0,37 a$ & $8,17 \pm 0,50 a$ \\
\hline & SG & $7,76 \pm 0,16 a$ & $7,89 \pm 0,19 a$ & $7,84 \pm 0,10 a$ & $7,89 \pm 0,19 a$ & $7,82 \pm 0,09 a$ \\
\hline \multirow{2}{*}{$\mathrm{CE}^{3}\left(\mathrm{dS} \cdot \mathrm{m}^{-1}\right)$} & SA & $11,45 \pm 5,79 a$ & $13,53 \pm 4,53 a$ & $17,19 \pm 5,12 a$ & $17,58 \pm 3,90 \mathrm{a}$ & $18,85 \pm 4,86 a$ \\
\hline & SG & $9,35 \pm 5,56 a$ & $12,48 \pm 6,05 a$ & $14,36 \pm 4,04 a$ & $11,98 \pm 5,48 a$ & $14,91 \pm 3,42 a$ \\
\hline
\end{tabular}

Referencias: ${ }^{1} \mathrm{MO}$ : Materia orgánica; ${ }^{2} \mathrm{C} / \mathrm{N}$ : Relación carbono: nitrógeno; ${ }^{3} \mathrm{CE}$ : Conductividad eléctrica.

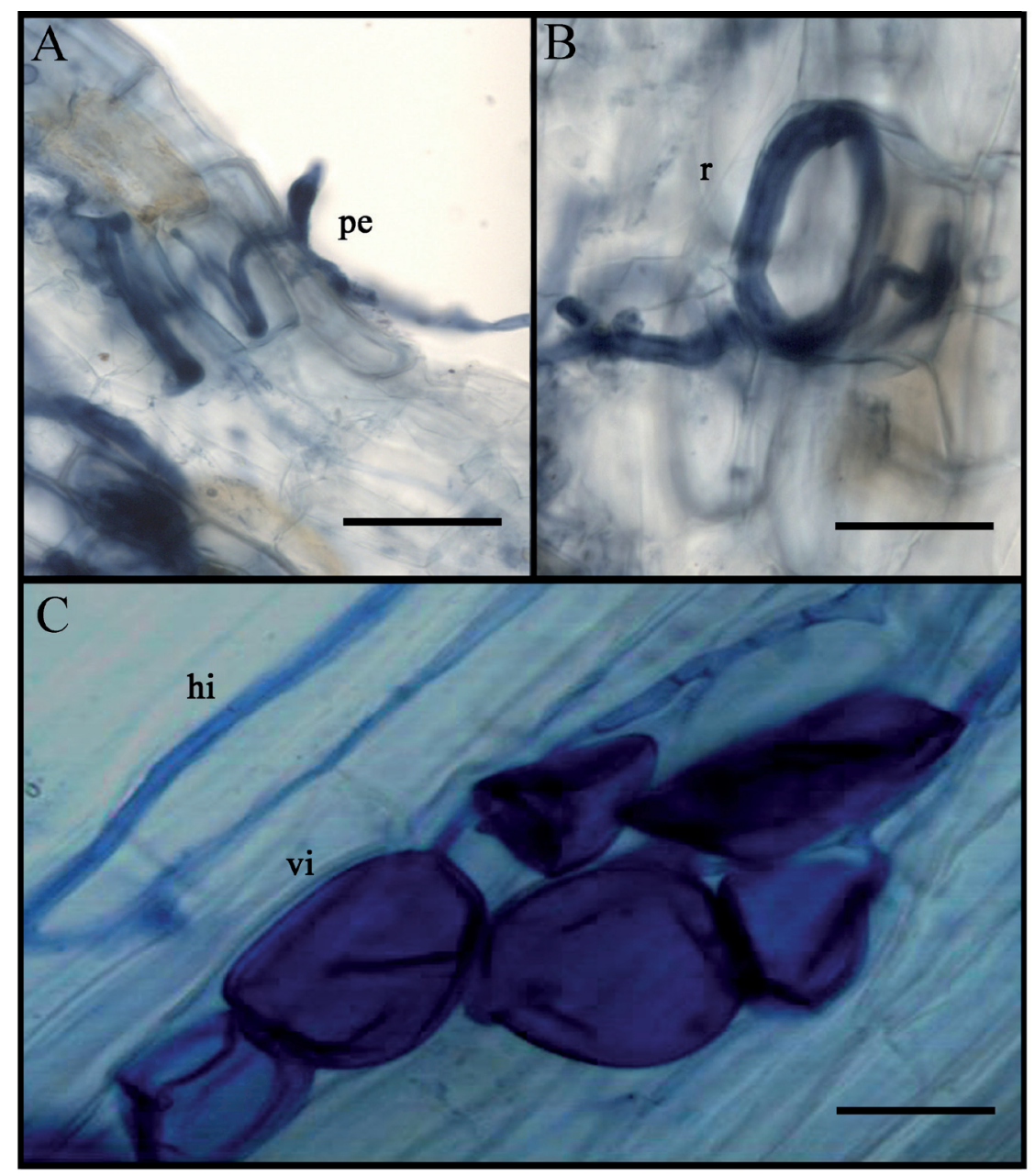

Fig. 1. Raíces colonizadas de Atriplex lampa. A: Punto de entrada (pe). B: Circunvolución intracelular (r). C: Hifas intracelulares (hi) y vesículas intracelulares (vi). Escala: $50 \mu \mathrm{m}$. 
Tabla 2. Colonización micorrícica (\%) en las

Salinas de Ambargasta (SA) y las Salinas

Grandes (SG) durante las estaciones húmeda

y seca. Resultados del análisis de la varianza

(ANOVA) de medidas repetidas. En negrita se

resaltan los valores de $P$ significativos.

\begin{tabular}{|lccc|}
\hline \multicolumn{1}{|c}{ Factor } & $\begin{array}{c}\text { Grados de } \\
\text { libertad }\end{array}$ & F & $\boldsymbol{P}$ \\
\hline profundidad & 4 & 1,29 & 0,28 \\
estación & 1 & 0,97 & 0,34 \\
sitio & 1 & 15,99 & $\mathbf{0 , 0 0 1}$ \\
estación x profundidad & 4 & 1,15 & 0,34 \\
sitio x profundidad & 4 & 0,55 & 0,7 \\
estación x sitio & 1 & 2,14 & 0,16 \\
$\begin{array}{l}\text { estación x sitio } \\
\text { x profundidad }\end{array}$ & 4 & 2,46 & 0,06 \\
\hline
\end{tabular}

HMA, a través de las circunvoluciones e hifas intracelulares. O bien, si hubiera arbúsculos, su ausencia en las raíces observadas podría deberse a que estas estructuras efímeras se generan durante períodos cortos y de máxima adquisición de nutrientes (Allen, 1983; Allen et al., 1989), siendo la probabilidad de encontrarlos muy baja (Brundrett et al., 1996).

Atriplex lampa presentó un alto porcentaje de colonización $(4,76 \%$ a $82,35 \%)$ en comparación con otras halófitas estudiadas, cuyos valores varían de 0,7 a 5,1\% (Dhillion et al., 1995; Landwehr et al., 2002; Wang et al., 2004; Tian et al., 2006). La capacidad de las plantas de volverse altamente colonizadas es una de las características más importantes en los ambientes semiáridos, ya que en respuesta a estas condiciones podrían presentar mayor dependencia micorrícica (Tao \& Zhiwei, 2005). En general, altos valores de $\mathrm{pH}$, de salinidad y los nutrientes del suelo (principalmente alta concentración de fósforo), disminuyen la colonización (Abbott \& Robson, 1984). Un menor porcentaje de colonización de $A$. lampa se observó en las SA, cuyos suelos presentaron mayores valores de $\mathrm{pH}$ (fuertemente alcalinos), y salinidad (mayor conductividad eléctrica) que los suelos de las SG.

A diferencia de lo observado en otros trabajos (Rillig \& Field, 2003; An et al., 1990), pero en concordancia con lo reportado por Ingleby et al. (1997), la concentración radical en A. lampa disminuyó significativamente con la profundidad del suelo y la colonización micorrícica fue similar en todo el perfil del suelo estudiado. Además,
Tabla 3. Concentración radical (número de segmentos radicales de $1 \mathrm{~cm} / 100 \mathrm{~cm}^{3}$ suelo) en las Salinas de Ambargasta (SA) y las Salinas Grandes (SG) durante las estaciones húmeda y seca. Resultados del análisis multivariado de la varianza (MANOVA). Para esta variable no fue posible aplicar un análisis de la varianza (ANOVA) de medidas repetidas, ya que las medidas no fueron independientes según el test de esfericidad de Mauchley $\left(X^{2}=65,38 ; P<0.001\right)$. En negrita se resaltan los valores de $P$ significativos.

\begin{tabular}{|lccc|}
\hline \multicolumn{1}{|c}{ Factor } & $\begin{array}{c}\text { Grados de } \\
\text { libertad }\end{array}$ & $\begin{array}{c}\text { Lambda } \\
\text { de Wilks }\end{array}$ & $\boldsymbol{P}$ \\
\hline $\begin{array}{l}\text { estación x } \\
\text { profundidad } \\
\text { sitio x profundidad }\end{array}$ & 4 & 0,37 & $\mathbf{0 , 0 0 8}$ \\
$\begin{array}{l}\text { estación x sitio } \\
\text { x profundidad }\end{array}$ & 4 & 0,74 & 0,39 \\
\hline
\end{tabular}

la concentración radical no se correlacionó significativamente con la colonización micorrícica. Una posible explicación de estos resultados, es que los propágulos infectivos estarían presentes en todas las profundidades permitiéndole al hospedante el acceso al agua y a los nutrientes en todo el perfil del suelo (Smith \& Read, 2008).

La presencia de las morfoespecies Funneliformis geosporum y F. mosseae, ya ha sido descripta para otros ambientes semiáridos del mundo (Sengupta \& Chaudhuri, 1990; Brown \& Bledsoe, 1996; Aliasgharzadeh et al., 2001; Carvalho et al., 2001; Hildebrandt et al., 2001). En este trabajo el género más comúnmente encontrado fue Glomus spp., que probablemente esté más adaptado a las condiciones de los sitios muestreados, ya que al igual que las plantas, los HMA podrían desarrollar estrategias adaptativas en ambientes estresantes (Carvalho et al., 2001; Landwehr et al., 2002; Soteras et al., 2012).

Este trabajo describe la presencia de HMA en la rizosfera de A. lampa y su colonización hasta los 50 $\mathrm{cm}$ de profundidad del suelo, durante dos estaciones y en dos ambientes salinos del norte de la provincia de Córdoba. A pesar de que esta especie pertenece a la familia Chenopodiaceae, usualmente registrada como no micorrícica, este es el segundo registro en Argentina sobre la presencia de HMA en raíces de $A$. lampa (Fontenla et al., 2001). Es necesario realizar estudios que evalúen la respuesta de $A$. lampa frente a la inoculación con HMA para confirmar la funcionalidad de la simbiosis, ya que en este trabajo 
๑ Estación húmeda $\square$ Estación seca
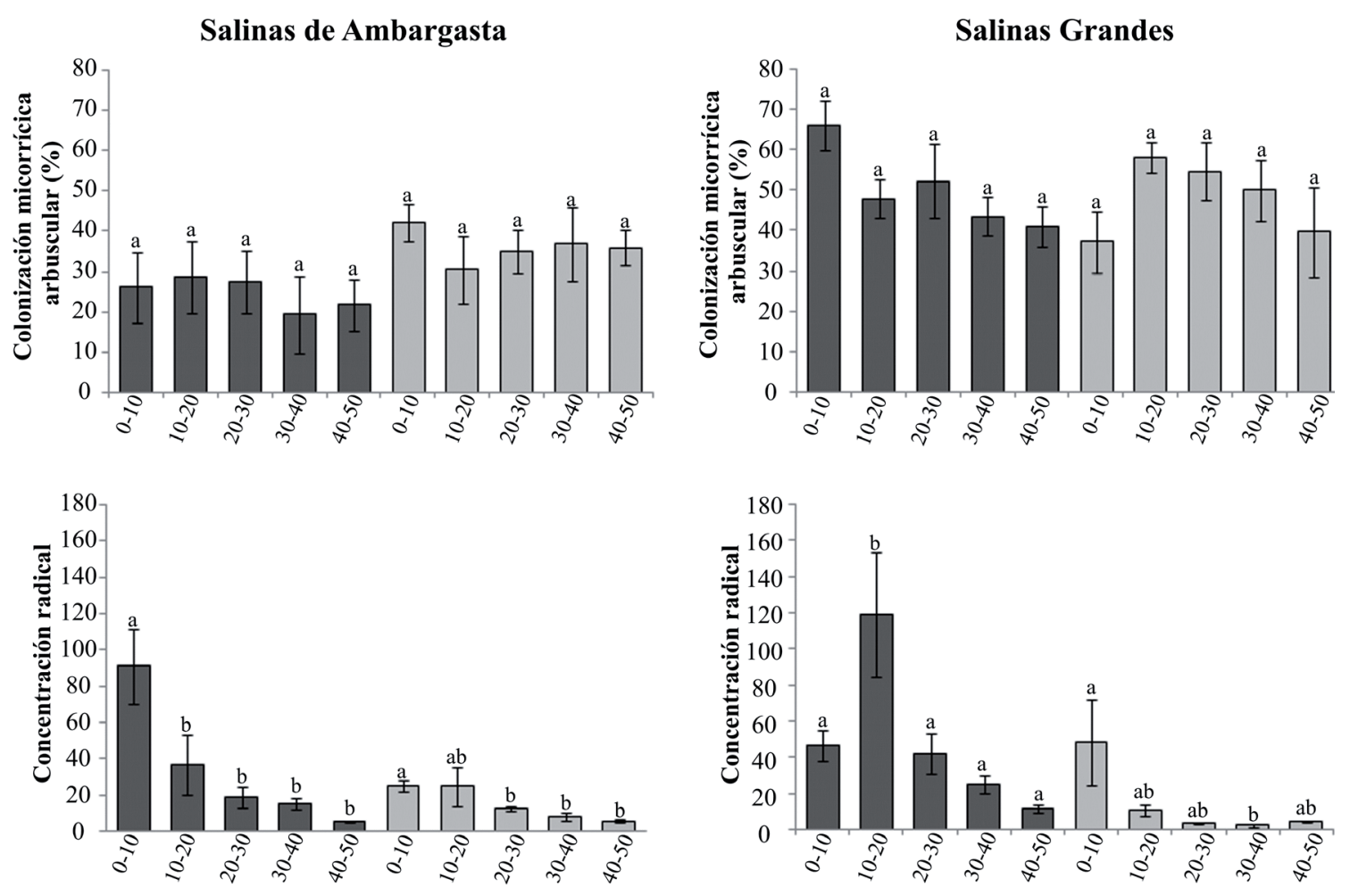

Profundidad del suelo (cm)

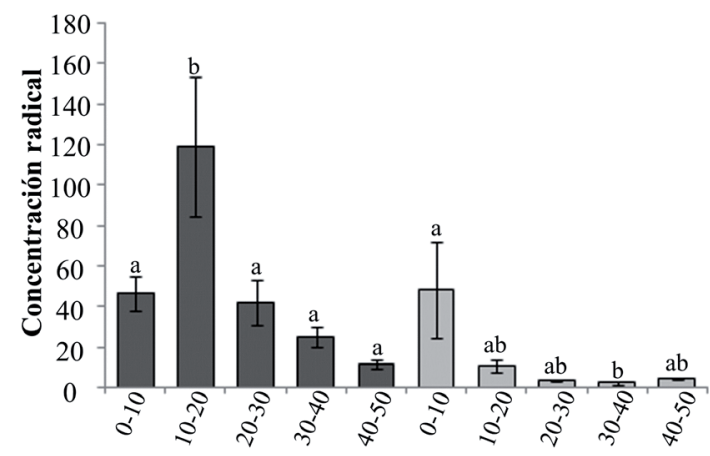

Profundidad del suelo (cm)

Fig. 2. Colonización micorrícica (\%) y concentración radical (número de segmentos radicales de $1 \mathrm{~cm} / 100$ $\mathrm{cm}^{3}$ suelo) de Atriplex lampa en las Salinas de Ambargasta y las Salinas Grandes durante las estaciones húmeda y seca, en las cinco profundidades de suelo $(0-10 \mathrm{~cm}, 10-20 \mathrm{~cm}, 20-30 \mathrm{~cm}, 30-40 \mathrm{~cm}$ y $40-50 \mathrm{~cm})$. Los valores son la media de 5 muestras \pm error estándar. En cada localidad, dentro de cada estación, las letras diferentes indican diferencias significativas según el test de Tukey HSD para un $P<0,05$.

no se observaron arbúsculos. Considerando que los suelos salinos de Córdoba ocupan el $14 \%$ de su superficie y que son ecosistemas poco estudiados, es importante profundizar el conocimiento de los microorganismos edáficos nativos en estos ambientes estresantes, y en particular de los HMA, por su relevancia en los procesos esenciales plantasuelo (Smith \& Read, 2008).

\section{Agradecimientos}

Este trabajo estuvo financiado por la Secretaría de Ciencia y Técnica -Universidad Nacional de Córdoba- y PICT 438-2006. Los autores agradecen a
L. S. Dominguez, L. Hernandez-Caffot, S. Longo y E. Nouhra por su ayuda en trabajos de campo. F.S. y N.C. agradecen al Consejo Nacional de Investigaciones Científicas y Técnicas (CONICET) y a la Agencia Nacional de Promoción Científica y Tecnológica por proveer sus respectivas becas. A.B. es investigadora de CONICET y M.C. es investigadora de CIC.

\section{Bibliografía}

ABBOTT, L. K. \& A. D. ROBSON. 1984. Colonization of the root systems of subterranean clover by three species of vesicular-arbuscular mycorrhizal fungi. New Phytol. 96: 275-278. 
AGUILERA, L. E., J. R. GUTIERREZ \& R. J. MORENO. 1998. Vesiculo arbuscular mycorrhizae associated with saltbushes Atriplex spp. (Chenopodiaceae) in the Chilean Arid Zone. Rev. Chil. Hist. Nat. 71: 291-302.

ALIASGHARZADEH, N., N. SALEH RASTIN, H. TOWFIGHI \& A. ALIZADEH. 2001. Occurrence of vesicular arbuscular mycorrhizal fungi in saline soils of the Tabriz Plain of Iran in relation to some pysical and chemical properties of soil. Mycorrhiza 11: $119-122$.

ALLEN, M. F. 1983. Formation of vesicular arbuscular mycorrhizae in Atriplex gardneri (Chenopodiaceae): seasonal response in a cold desert. Mycologia 75: 773-776.

ALLEN, M. F., E. B. ALLEN \& C. F. FRIESE. 1989. Responses of the non-mycotrophic plant Salsola kali to invasion by vesicular-arbuscular mycorrhizal fungi. New Phytol. 111: 45-49.

ALLEN, M. F. \& E. B. ALLEN. 1990. Carbon source of VA mycorrhizal fungi associated with Chenopodiaceae from a semiarid shrub steppe. Ecology 71: 2019-2021.

AN, Z. Q., J. H. GROVE, J. W. HENDRIX, D. E. HERSHMAN \& G. T. HENSON. 1990. Vertical distribution of endogonaceous mycorrhizal fungi associated with soybean, as affected by soil fumigation. Soil Biol. Biochem. 22: 715-719.

BREMNER, J. M. \& C. S. MULVANEY. 1982. Nitrogentotal. In: PAGE, A. L. (ed.), Methods of soil analysis Part II, pp. 595-562. American Society of Agronomy: Soil Science Society of America, Madison, Wisconsin.

BROWN, A. M. \& C. BLEDSOE. 1996. Spatial and temporal dynamics of mycorrhizas in Jaumea carnosa, a tidal saltmarsh halophyte. J. Ecol. 84: 703-715.

BRUNDRETT, M. 1991. Mycorrhizas in natural ecosystems. In: MACFAYDEN, A., M. BEGON \& A. H. FITTER (eds.), Advances in Ecological Research, vol. 21, pp. 171-262. Academic Press London.

BRUNDRETT, M., N. BOUGHER, B. DELL, T. GROVE \& N. MALAJCZUK. 1996. Working with Mycorrhizas in Forestry Agriculture. ACIAR Monograph 32, Canberra, Australis: Australian Centre for International Agricultural Research.

CABIDO, M. \& M. ZAK. 1999. Vegetación del Norte de Córdoba. Córdoba: Secretaría de Agricultura, Ganadería y Recursos Renovables de Córdoba.

CABIDO, M., C. ROSACHER, J. M. ROQUÉ GARZÓN, D. CABIDO, J. GORGAS \& J. PIANA. 2006. Reserva de Usos Múltiples Salinas Grandes. En: CABIDO, M., C. ROSACHER, J. M. ROQUÉ GARZÓN \& D. CABIDO (eds.), Áreas Naturales Protegidas de la Provincia de Córdoba, pp. 81-96. Agencia Córdoba Ambiente, Gobierno de Córdoba, La Voz del Interior, Córdoba.

CARVALHO, L. M., I. CAÇADOR \& M . A. MARTINS-
LOUÇÃO. 2001. Temporal and spatial variation of arbuscular mycorrhizas in salt marsh plants of the Tagus estuary (Portugal). Mycorrhiza 11: 303-309.

COFRÉ, N., A. BECERRA, E. NOUHRA \& F. SOTERAS. 2012. Arbuscular mycorrhizae and dark-septate endophytes on Atriplex cordobensis in saline sites from Argentina. J. Agric. Technol. (en prensa).

DHILLION, S. S., P. E. VIDIELLA, L. E. AQUILERA, C. F. FRIESE, E. DE LEON, J. J. ARMESTO \& J. C. ZAK. 1995. Mycorrhizal plants and fungi in the fog-free Pacific coastal desert of Chile. Mycorrhiza 5: 381-386.

FONTENLA, S., E. CHAIA, C. BUSTOS \& A. PELLIZA. 2001. Microorganismos simbióticos en Atriplex. Bol. Soc. Arg. Bot. 36: 114.

FÜZY, A., B. BIRÓ, T. TÓTH, U. HILDEBRANDT \& H. BOTHE. 2008. Drought, but not salinity, determines apparent effectiveness of halophytes colonized by arbuscular mycorrhizal fungi. J. Plant Physiol. 165: 1181-1192.

GERDEMANN, J. W. \& T. H. NICOLSON. 1963. Spores of a mycorrhizal Endogone species extracted from the soil by wet sieving and decanting. Trans. Br. Mycol. Soc. 46: 235-244.

GIOVANNETTI, M. \& B. MOSSE. 1980. An evaluation of techniques for measuring vesicular-arbuscular mycorrhizal infection in roots. New Phytol. 84: 489500 .

HILDEBRANDT, U., K. JANETTA, O. FOUAD, B. RENNE, K. NAWRATH \& H. BOTHE. 2001. Arbuscular mycorrhizal colonization of halophytes in Central European salt marshes. Mycorrhiza 10: 175-183.

HIRREL, M. C., H. MEHRAVARAN \& J. W. GERDEMANN. 1978. Vesicular-arbuscular mycorrhizae in the Chenopodiaceae and Cruciferae: do they occur? Can. J. Bot. 56: 2813-2817.

INGLEBY, K., O. DIAGNE, J. D. DEANS, D. K. LINDLEY, M. NEYRA \& M. DUCOUSSO. 1997. Distribution of roots, arbuscular mycorrhizal colonisation and spores around fast-growing tree species in Senegal. For. Ecol. Manag. 90: 19-27.

INVAM (International Collection of Vesicular-Arbuscular Mycorrhizal Fungi). Disponible en http://invam.caf. wvu.edu/Myc_Info/Taxonomy/species.htm [Acceso: Septiembre 2008].

JACKSON, M. L. 1964. Análisis químico de suelos. 2nd Edn. Omega, Barcelona.

JARSÚN, B., J. A. GORGAS, E. ZAMORA, E. BOSNERO, E. LOVERO, A. RAVELO \& J. L. TASSILE. 2003. Recursos Naturales de La Provincia de Córdoba: Los Suelos. Nivel de Reconocimiento 1:500.000. Argentina. Agencia Córdoba D.A.C. y T. S.E.M Dirección de Ambiente e INTA (INSTITUTO NACIONAL DE TECNOLOGÍA 


\section{F. Soteras et al. - Glomeromycota: presencia y colonización radical en Atriplex lampa}

AGROPECUARIA-MANFREDI).

KABIR, Z., I. P. O'HALLORAN, P. WIDDEN \& E. HAMEL. 1998. Vertical distribution of arbuscular mycorrhizal fungi under corn (Sea mays L.) in notill and conventional tillage systems. Mycorrhiza 8: 53-55.

KHAN, M. A. \& N. C. DUKE. 2001. Halophytes - A resource for the future. Wetl. Ecol. Manag. 6: 455456.

KRÜGER, M., C. KRÜGER, C. WALKER, H. STOCKINGER, A. SCHÜßLER. 2012. Phylogenetic reference data for systematics and phylotaxonomy of arbuscular mycorrhizal fungi from phylum to species level. New Phytol. 193:970-84.

LANDWEHR, M., U. HIDELBRANDT, P. WILDE, K. NAWRATH, T. TOTH, B. BIRO \& H. BOTHE. 2002. The arbuscular mycorrhizal fungus Glomus geosporum in European saline, sodic and gypsum soils. Mycorrhiza 12: 199-211.

LUGO, M. A., M. E. GONZÁLEZ MAZA \& M. N. CABELLO. 2003. Arbuscular mycorrhizal fungi in a mountain grassland II: Seasonal variation of colonization studied along its relation to grazing and metabolic host type. Mycologia 93: 407-415.

NELSON, D. W. \& L. E SOMMERS. 1982. Total carbon, organic carbon, and organic matter. In: PAGE, A. L., R. H. MILLER \& D. R. KEENEY (eds.), Methods of soil analysis, Part 2, pp. 639-577. Amer. Soc. Agron., Inc., Madison WI.

OCAMPO, J. A., J. MARTÍN \& D. S. HAYMAN. 1980. Influence of plant interactions on vesicular-arbuscular mycorrhizal infection I. Host and non-host plants grown together. New Phytol. 84: 27-35.

OEHL, F., E. SIEVERDING, J. PALENZUELA, K. INEICHEN \& G. A. DA SILVA. 2011. Advances in Glomeromycota taxonomy and classification. IMA Fungus 2: 191-199.

OMAR, M. B., L. BOLLAND \& W. A. HEATHER. 1979. P.V.A. (polivinil alcohol). A permanent mounting medium for fungi. Bull. Br. Mycol. Soc. 13: 31-32.

PASSERA, C. B. \& O. BORSETTO. 1989. Aspectos ecológicos de Atriplex lampa. Invest. Agrar: Prod. Prot. Veg. 4: 179-198.

PETERSON, R. L., H. B. MASSICOTTE \& L. H. MELVILLE. 2004. Arbuscular mycorrhizas. In: CAVERS, P. B. (ed.), Mycorrhizas: Antomy and Cell Biology, pp. 55-79. National Research Council of Canada, Ottawa.

PHILLIPS, J. M. \& D. S. HAYMAN. 1970. Improved procedures for clearing roots and staining parasitic and vesicular-arbuscular mycorrhizal fungi for rapid assessment of infection. Trans. Br. Mycol. Soc. 55: 158-161.

PLENCHETTE, C. \& R. DUPONNOIS. 2005. Growth response of the saltbush Atriplex nummularia L. to inoculation with the arbuscular mycorrhizal fungus Glomus intraradices. J. Arid Environ. 61: 535-540.

RILLIG, M. C. \& C. B. FIELD, 2003. Arbuscular mycorrhizae respond to plants exposed to elevated atmospheric $\mathrm{CO}_{2}$ as a function of soil depth. Plant Soil 254: 383-391.

RUIZ-LOZANO, J. M., R. AZCÓN \& M. GOMEZ. 1996. Alleviation of salt stress by arbuscular mycorrhizal Glomus species in Lactuca sativa plants. Plant Physiol. 98: 767-772.

SCHENCK, N. C. \& Y. PEREZ. 1990. Manual of identification of vesicular-arbuscular mycorrhizal fungi. INVAM, University of Florida, Gainesville.

SENGUPTA, A. \& S. CHAUDHURI. 1990. Vesicular arbuscular mycorrhiza (VAM) in pioneer salt marsh plants of the Ganges River delta in West Bengal (India). Plant Soil 122: 111-113.

SMITH, S. E. \& D. J. READ. 2008. Mycorrhizal Symbiosis. 3rd ed. Academic Press, Elsevier, Amsterdam.

SMITH, S. E. \& F. A. SMITH. 2011. Roles of Arbuscular Mycorrhizas in Plant Nutrition and Growth: New Paradigms from Cellular to Ecosystems Scales. Annu. Rev. Plant Biol. 62: 227-250.

STATISTICA: software platform of StatSoft. Disponible en: http://www.statsoft.com/ [Acceso: Diciembre 2008].

SOTERAS, F., A. BECERRA, N. COFRÉ, J. BARTOLONI \& M. CABELLO. 2012. Arbuscular mycorrhizal fungal species in saline environments of Central Argentina: seasonal variation and distribution of spores at different soil depths. Sydowia (en prensa).

TAO, L. \& Z. ZHIWEI. 2005. Arbuscular mycorrhizas in a hot and arid ecosystem in southwest China. Appl. Soil Ecol. 29: 135-141.

TIAN, C., Z. SHI, Z. CHEN \& G. FENG. 2006. Arbuscular mycorrhizal associations in the Gurbantunggut Desert. Chin. Sci. Bull. 51: 140-146.

WALKER, C., W. MIZE \& H. S. MCNABB. 1982. Populations of endogonaceous fungi at two populations in central Iowa. Can. J. Bot. 60: 25182529.

WANG, F. Y., R. J. LIU, X. G. LIN \& J. M. ZHOU. 2004. Arbuscular mycorrhizal status of wild plants in salinealkaline soils of the Yellow River Delta. Mycorrhiza 14: 133-137.

ZANGARO, W., S. M.A. NISIZAKI, J. C. B. DOMINGOS, E. M. NAKANO. 2002. Arbuscular mycorrhizal in native woody species of Tibagi River Basin, Paraná. Cerne 8: 77-87.

Recibido el 25 de junio de 2012, aceptado el 23 de noviembre de 2012. 
